\title{
A systematic review of approaches to modelling lower limb muscle forces during gait: applicability to clinical gait analyses.
}

\author{
Ursula Tinler $^{\mathrm{a}, \mathrm{b}}$, Kristen Hollands ${ }^{\mathrm{a}}$, Richard Jones ${ }^{\mathrm{a}}$, Richard Baker ${ }^{\mathrm{a}}$
}

${ }^{a}$ University of Salford, School of Health Science, Allerton Building, Frederick Road Campus, Salford, M6 6PU, United Kingdom

${ }^{\mathrm{b}}$ BG Unfallklinik Ludwigshafen, Zentrum für Bewegungsanalytik, Forschung und Lehre, Ludwig-Guttmann Straße 13, 67071 Ludwigshafen, Germany

Corresponding author:

Ursula Trinler

Forschung und Lehre

BG Unfallklinik Ludwigshafen

Ludwig-Guttmann Str. 13

67071 Ludwigshafen

Germany

Ursula.trinler@bgu-ludwigshafen.de,0049-621 68108498 


\begin{abstract}
Computational methods to estimate muscle forces during walking are becoming more common in biomechanical research but not yet in clinical gait analysis. This systematic review aims to identify the current state-of-the-art, examine the differences between approaches, and consider applicability of the current approaches in clinical gait analysis.
\end{abstract}

A systematic database search identified studies including estimated muscle force profiles of the lower limb during healthy walking. These were rated for quality and the muscle force profiles digitised for comparison.

From 13.449 identified studies, 22 were finally included which used four modelling approaches: static optimisation, enhanced static optimisation, forward dynamics and EMGdriven. These used a range of different musculoskeletal models, muscle-tendon characteristics and cost functions. There is visually broad agreement between and within approaches about when muscles are active throughout the gait cycle. There remain considerable differences (CV $7 \%$ to $151 \%$, range of timing of peak forces in gait cycle $1 \%$ to $31 \%$ ) in patterns and magnitudes of force between and within modelling approaches.

The main source of this variability is not clear. Different musculoskeletal models, experimental protocols, and modelling approaches will clearly have an effect as will the variability of joint kinetics between healthy individuals. Limited validation of modelling approaches, particularly at the level of individual participants, makes it difficult to conclude if any of the approaches give consistently better estimates than others.

While muscle force modelling has clear potential to enhance clinical gait analyses future $\underline{\text { research is needed to improve validation, accuracy and feasibility of implementation in }}$ clinical practice. 


\section{Keywords}

modelling approaches, muscle forces, gait, lower limb. 


\section{INTRODUCTION}

Knowing the force profiles of individual muscles during walking may help to better identify various neuro-musculoskeletal impairments from clinical gait analysis measurements and give a better understanding of how these affect walking. Patellar femoral pain, for example is often thought [1] to be due to an imbalance of force between agonist and antagonists and can lead to an excessive loading of knee joint and subsequent risk of developing degenerative joint conditions and injuries [2]. A better understanding of actual muscle forces in this condition, and many others, might help to identify the exact mechanism of gait impairments. Whilst muscle force estimation is becoming more and more common in biomechanical research, it is still not used routinely in a clinical context.

A variety of computational techniques are now available to estimate muscle forces [3]. These modelling approaches have already been applied in a variety of research studies related to sport performance or clinical interventions [4-6]. Use of muscle force modelling has, however, not yet become established in a routine clinical gait analysis, despite that its potential utility was highlighted ten years ago [7]. A range of models is available incorporating different aspects of musculoskeletal morphology, kinematics and kinetics and

muscle function based on a range of different assumptions [3, 5, 8]. Different cost functions have been applied to optimise force estimation and different models require different experimental inputs (kinematics, kinetics, EMG etc.). Each of these variations in model assumptions, optimisation techniques and input data have their own inherent limitations which may yield different force estimations and make it difficult for users in clinical gait analysis to select the most appropriate technique. However, the consistency between these different modelling techniques and the feasibility of using a modelling approach to estimate muscle forces within clinical gait analysis has not yet been investigated. 
To establish the potential feasibility and utility of muscle force modelling in clinical movement analysis a systematic review to compare the range of approaches is needed. This will allow identification of differences between models that cause differences in outputs and facilitate the development of a standardised protocol for the use of muscle force modelling which can be applied in a routine Clinical Gait Analysis (CGA). Therefore, the objective of this review is to facilitate a description of the state-of-the-art in muscle force estimation in gait analysis. Muscle force data will be extracted and pooled within the different modelling approaches identified in order to determine the consensus amongst different studies using the same modelling approach but also between different modelling approaches.

The aims of the systematic review can, therefore, be summarised as follows:

1. Identification of different techniques to estimate muscle forces in studies related to healthy human walking.

2. Examination of muscle force profiles during walking and the consistency of these within and between different modelling approaches.

3. Consideration of the applicability of current muscle force estimation approaches $\underline{\text { to clinical practice and discussion of what may be required for future research to }}$ establish implementation in clinical gait analysis."

At the same time, as kinetic information is used into the muscle force estimation process, a parallel systematic review of papers reporting joint moments was conducted. This will be described in the Methodology below but the results are presented separately in an Appendix.

\section{METHODS}

Search and selection strategy 
To locate relevant papers, the following scientific databases were used (Jan 1990 - Dec 2016):

- Ovid including Medline and AMED.

- $\quad$ EBSCO, including CINHAL and SPORTDiscus.

- Web of Knowledge.

The start of the search period was set to 1990 which was when the first standardised musculoskeletal models which could be applied during walking were developed $[9,10]$.

A free keyword search was undertaken with additional Medical Subject Headings (MeSH)

[11]. The following search terms and their synonyms were included: gait, lower limb, moment, muscle, forces, mathematical model, modelling approaches, computer simulation, EMG driven, static optimisation, forward dynamics, dynamic optimisation, musculoskeletal model, and inverse dynamics. Furthermore, the bibliographies of identified papers were manually scanned to identify missing but relevant studies.

Two independent reviewers (UT, RB) first screened titles and abstracts to exclude obviously irrelevant papers and then reviewed full text versions of the remaining paper to assess whether papers met the eligibility criteria listed below. Any disagreements between reviewers where resolved through discussion. Figure 1 presents a flow chart of the selection procedure.

\section{Eligibility criteria}

\section{Participants}

Studies were included if they involved healthy adult participants ( $>18$ years). Studies which included healthy participants as a control group for comparison with another patient group were included but only data from those healthy controls were extracted. The maximum age was set to 60 years as walking kinematics [12] and muscle force capacity [13, 14] is understood to change beyond this age. 
Studies reporting estimates of muscle forces for individual healthy humans calculated from data captured during overground walking were included. Owing to ongoing debate as to the similarity of treadmill and overground walking $[15,16]$ only studies reporting estimates of muscle forces for individual healthy humans calculated from data captured during overground walking in a laboratory setting were included. Therefore, treadmill walking as well as studies of movements other than walking in environments other than a movement laboratory were excluded. The following muscles were identified as important in human walking $[17,18]$ and are included in data extraction: soleus, gastrocnemius, tibialis anterior, rectus femoris, vastii muscle group, hamstrings, gluteus maximus and medius, and iliopsoas.

Studies presenting predictive simulations not based on data from individuals were excluded as clinical gait analysis requires models to be based on real experimental data. Gait analyses on a treadmill were not accepted as it may have changed the participants' walking biomechanics [15]. Studies were only included if experimental data, joint kinematics and kinetics data and/or EMG activations had been captured within the researchers' laboratory (no experimental data from external laboratories or previously published databases). If two or more modelling approaches were compared in any study then results from all modelling approaches were included.

\section{Outcome measures}

In order to facilitate the objective of comparing time varying muscle force estimations based on different modelling approaches, only studies providing graphical or numerical data of time varying muscle force estimations over the entire gait cycle or a defined part of the gait cycle were included. 


\section{Quality assessment tool and data synthesis}

A customised quality assessment tool was applied to rate and describe the quality of methodology and reporting of the musculoskeletal model and simulation. This was carried out independently by the two reviewers (UT, RB). Discrepancies in quality ratings between reviewers were settled by consensus in discussion.

The systematic quality assessment procedure was designed by combining items from quality assessment tools for kinematic studies [19], randomised and non-randomised clinical interventions $[20,21]$ and additional items specific to muscle modelling approaches. Included studies were thus assessed on twelve aspects relating to the reported methodological quality, the research design of the study, the description of the experimental data collection and processing, the explanation of applied musculoskeletal models and modelling approaches, and the validation and discussion of limitations of the study. Some of these items were further broken down into sub-items. A full list of the items can be found in the electronical appendix (Figure S1). The score of an item and its sub-items were summed and transformed in a percentage of the maximum possible score. The overall score was then taken as the average of these relative scores allowing the highest score to be $100 \%$, and the lowest to be $0 \%$.

Data extraction involved a graphical synthesis of all the muscle force patterns reported in the included studies. Estimated muscle force profiles were extracted and automatically digitised by the programme GetData Graph Digitiser (version 2.26, Sergei Fedorov, 2013, Russia). Accuracy of digitalisation was assessed by digitising our own experimental data three times. No appreciable differences (less than $1 \%$ of maximum value) between digitalisation and raw data were found. In the case that automatic digitalisation failed due to the quality of the graph, the graph was digitised by the operator manually selecting points on the graph. For those papers which focused exclusively on the stance phase of walking without stating the stanceto-swing ratio of the gait cycle a 60\%:40\% ratio was assumed [22]. 
Digitised graphs were further analysed in Matlab (R2012b) by normalising them to the body weight of the participants of the study to account for influences occurring through different body sizes [23]. In case the specification of the participants' body mass was missing the averaged body mass across all studies was used for normalisation. All muscle force profiles were averaged across studies to gain an overall mean at each time point in the gait cycle as well as one and two standard deviation bands. In cases when estimation of muscle force was reported separately for different muscle compartments (e.g., gastrocnemius lateralis and medialis, psoas and illiacus, vastus medialis and lateralis, hamstrings compartments) the data reported were summed up across compartments to generate a force profile for the whole muscle (gastrocnemius, iliopsoas, vastii, hamstrings).

The grand mean $(\mu)$ of all studies will be presented and discussed as well as its standard deviation $(\sigma)$ to describe the variability between studies. To facilitate comparisons between muscles generating different magnitudes of force, the coefficient of variation $(\mathrm{CV})$

$$
C V=\frac{\sigma}{\mu} * 100
$$

is also calculated for specific peak values, as has been done before for similar purposes [24].

\section{RESULTS}

Figure 1 describes the development of the systematic review process. After the reviewing process 22 from 9870 originally identified studies were eligible for inclusion on the basis of presenting time varying data (graphically or numerically) of muscle force estimation over the gait cycle.

The characteristics of included studies are detailed in Table 1. All but six studies were based on a single participant, while $80 \%$ of participants were male. Three studies did not give any 
information about the participants' characteristics [25-27], and further three gave only partial details [28-30]. In general, participants were asked to walk at their self-selected speed. Only nine out of all 19 studies, however, reported the actual walking speed (one other study defined cadence in steps/min [31]). Eight studies focused on the stance phase only which lasted, on average, $60 \%$ of the gait cycle. White and Winter [29] defined the gait cycle from toe off to toe off.

Included studies broadly fell into four categories of modelling approaches, which are inverse dynamics (static optimisation SO, 14 studies), forward dynamics (FD, three studies), EMGdriven models (six studies) and static optimisation combined with a dynamic optimisation criteria (enhanced-SO, four studies).

\section{Quality Appraisal of Included Studies}

Table 2 shows the quality assessment scores for each study. The overall quality with which studies were reported varied from $48 \%$ to $81 \%$. Enhanced static optimisation models and EMG-driven models reached an overall higher score (average of $74 \%$ and $70 \%$, respectively) compared to static optimisation (63\%) and forward dynamics (58\%) largely because these studies presented the experimental details in average more comprehensively. By contrast static optimisation and enhanced static optimisation studies tended to report their modelling approaches in more detail than the other two groups.

\section{Modelling techniques}

Static optimisation is an inverse dynamic technique. It solves the redundancy problem (more muscles spanning a joint than degrees of freedom exist) by dividing the experimental joint moments between the different muscles acting across the joint at each instant in time independently. The forward dynamics approaches identify the muscle activation pattern which led to the closest match of model and experimental kinematics over the whole gait cycle. 
EMG-driven models additionally use experimental EMG excitations as input in the estimations to define muscular activation patterns. Four studies presented an enhanced static optimisation methods called physiological inverse dynamics [26] and computed muscle control [3, 32, 33], both including, additionally, a time-dependent characteristic, all representing the enhanced-SO group.

Static, enhanced inverse and forward optimisation models used a variety of different optimisation functions to overcome the redundancy problem (Table 1). These functions minimise a specific cost function, which were either the sum of squared muscle activations, the sum of all muscle forces, the sum of (squared) muscle stress, or the minimisation of the metabolic energy (Table 1). EMG-driven studies incorporate matching of muscle activations to measured EMG signals as part of the cost function.

\section{Musculoskeletal models}

The most frequently used musculoskeletal models (twelve studies) were derived from the model of Delp and colleagues [34]. Each side consists of seven rigid bodies (pelvis, femur, patella, tibia/fibula, talus, foot, toes) with 43 musculotendon actuators acting between insertion and origins defined by digitising human bones. The actuators were Hill-type muscle models, adapted from Zajac's work [35], and scaled in its maximum isometric force, the optimal-muscle fibre length, the pennation angle of the muscle, and the tendon slack length. Except Besier et al. [36], papers using the model of Delp adjusted the number of segments or the number of muscle tendon actuators depending on their focus of the study. Some included an upper body model, mostly by one segment representing the head arms and trunk (HAT) or others incorporated additional segments within the foot.

Four studies [3, 8, 37, 38] used the musculoskeletal model of Anderson and Pandy [5] which is modelled as a 10-segment (HAT, pelvis, left and right thigh, left and right shank, left and 
right hind- and forefoot) 23 degree of freedom linked model actuated by 54 muscle tendon muscles (24 muscles per leg). Muscle-tendon paths were adapted from the Delp model and the dynamics of the muscle-tendon unit from Zajac [35]. Six studies developed their own models. They differ in the number of muscle-tendon actuators and in the number of segments. The geometric parameters of the segments are either extracted from studies on cadavers $[39,40]$, through modelling approaches [41], or through analysis on living tissue [42, 43].

\section{Validation of estimated muscle forces}

A direct validation of estimated muscle forces is only possible with in-vivo techniques. However, good quality EMG data indicate when a muscle should be active. 18 of the 22 muscle modelling studies compared their estimated muscle forces with EMG patterns. This was most often on the basis of on-off patterns of the muscle activity rather than on the whole profile of the EMG signal. The researchers rarely captured EMG data of their own participants and mostly used EMG patterns found in the literature. Only six studies compared the muscle force estimation to experimental EMG data that they had captured themselves. The other studies which used EMG as a validation tool included EMG excitations from Inman [44], Perry [45], Hof [46], or Anderson and Pandy [8]. Leardini and O’Connor [28] do not state their source of EMG patters used in their graph for validation. Three EMG-driven studies used experimental joint moments or power and compared them to estimated joint moments and powers calculated through the estimated muscle force estimations. Four studies did not validate their muscle force estimations at all.

\section{Outcome measures}

Four studies $[3,8,26,47]$ compared two or more modelling techniques with each other (see Table 1) which were all separately included into the digitising process. Results from two static optimisation approaches from Anderson and Pandy [8] (with and without inclusion of force- 
length-velocity curve) were averaged for inclusion in the analysis, as their "force-lengthvelocity properties of muscle had little influence on the static solutions" [8].

Table 3 describes maximum peak forces, its standard deviation and CV. The average force distribution across a gait cycle are presented in Figure 2. Note that studies which included EMG-driven models did not include force estimations for muscles acting on the hip (iliopsoas, gluteus maximus and medius).

In general, the shape of the estimated muscle force profiles show broad agreement between modelling approaches (Figure 2). For some of the muscles, however, differences exist. Forward dynamic solutions are particularly susceptible to sharp peaks and discontinuities leading to more variablity between model standard deviation profiles in the muscle force estimations than other techniques. There is broad agreement within and between approaches as to when muscles are not active but considerable variability in the estimated magnitude of muscle force when they are active.

Generally speaking, the EMG driven approaches show agreement especially with static optimisation and enhanced-SO but suggest smaller magnitudes of force and some residual activation of muscles at times when the other approaches suggest no activation. This is especially true for rectus femoris, the vasti, tibialis anterior and, to a lesser extent, soleus. An exception is that the EMG driven models suggest higher forces in the tibialis anterior during swing, which is confirmed by enhanced-SO. It is also interesting that forward driven models do not predict tibialis activation at all in first double support in contrast to the other approaches, while static optimisation has no pronounced second peak in mid swing. The standard deviation bars in Figure 2 show that, within individual modelling approaches, there is general agreement between studies as to when muscles are inactive. On the other hand, for all muscles where data is available from three or more static optimisation and 
forward dynamic models there is considerable variability in the magnitude of the forces during active periods. The enhanced-SO and EMG driven models appear to show more consistent estimates of muscle force. These findings are confirmed by the tabulated average and standard deviations of maximal force generation (Table 3). Most of the coefficients of variation are above $50 \%$ reflecting considerable variability in force estimates between models, even within approaches. Lowest CV values are presented in average for enhanced-SO with only two muscles exceeding $40 \%$ (first peak of rectus femoris, gluteus medius).

\section{DISCUSSION}

This is the first systematic synthesis of different computational methods to estimate muscle forces during walking, to examine the differences between approaches, and to consider their potential for use in clinical gait analysis. The study showed a broad agreement in when muscles are active between and within modelling approaches, however, with considerable differences between the estimated patterns and magnitudes of force generation.

\section{Quality of studies}

The quality rating of studies varies quite considerably. It is important to recognise that this primarily reflects the quality of how comprehensively the techniques and results have been reported and does not necessarily reflect the quality with which the research was conducted. A number of studies scored particularly badly $[8,27,48]$ because the focus on modelling issues led to omissions of key experimental details. The specific task and pre-modelling signal processing issues were particularly badly reported across all studies. All but five studies [3, $29,30]$, however, scored zero on at least one category. In such a complex area, and with few studies to identify which signals and parameters models are most sensitive to, it is important that all aspects of data collection and modelling are adequately reported. 


\section{Variability of outputs}

Broadly speaking, the different models agree in predictions as to when specific muscles are active within the gait cycle. There is considerable variability, however, in predictions of the peak muscle forces, with the coefficient of variation across studies typically considerably greater than $50 \%$. The source of this variability is not clear. The authors looked for evidence that papers with higher quality rating or published more recently showed more consistent results but this did not appear to be the case (noting, as in the paragraph above, that simply because a study has been reported well does not guarantee that it has been conducted well). Four studies have compared outputs of different models [3, 8, 26, 47]. Whilst Anderson and Pandy present convincing evidence that static optimisation and forward dynamics are "practically equivalent" [8] the other studies are less convincing. The study of Lin et al. [3] is particularly interesting in that three differing modelling techniques were applied with the same biomechanical model. Whilst the authors concluded that the patterns of muscle loading were "similar", substantial differences in both the pattern of activation and peak load values can be seen for at four of the eight muscles for which walking data is presented. (The study even shows quite substantial differences between two different implementations of exactly the same simulation).

Another potential contributor to variability in model outputs (particularly between different papers) is variability in model inputs. Data presented and discussed in the appendix suggests considerable variability between joint kinetics presented in different studies. Some of this may result from different analytical techniques but Pinzone et al. [49] have recently commented on the extent of inter-individual variability in joint kinetics even after using non-dimensional normalisation techniques to effectively remove systematic variation with leg length, mass or age. Most of the modelling studies reported in this paper have been based on one individual and differences between studies may well reflect, in part, differences in the individuals 
studied. Similarities in the average traces across studies within each of the modelling approches, despite large variability between studies, might reflect this. It would also explain why similarity between the EMG driven studies (which all averaged results across a number of individuals) appear more consistent than models in the other categories (which generally presented results from just one individual).

\section{Validation}

Given the variability between model outputs some sort of validation is required to distinguish which are actually succeeding [50]. Seven of the 22 studies made no attempt at validating outputs. A further four did so by comparison with data taken from the literature rather than the individuals modelled (which might be an important issue if there is considerable variability in joint kinetics between individuals as suggested above).

Validations are generally undertaken against EMG measurements. This is useful to give a broad indication of whether the estimated patterns of force generation are sensible. it is of limited use in validating the magnitude of the force estimates, given that there is no simple relationship between the EMG signal and the magnitude of force generation within the muscles [7]. Recent studies comparing joint contact loads with those measured by transducers [51] are more convincing but also more restricted in who they can be applied to. Given the potential importance of kinetic variability (and thus presumably EMG variability) between individuals, validation at the level of the individual is important and comparisons between different techniques should either be conducted on the same datasets or on a sufficient number of individuals for these differences to be averaged out.

Therefore, full quality scores were given when estimated muscle forces were compared against EMG measured parallel to the walking trial of interest. However, considering the non-linear relationship between EMG and muscle forces, validation against EMG 
may not be the best or only method to achieve clinical validation or implementation. Instead, validation against parameters like sensitivity of muscle force estimations to changes in the musculoskeletal system (caused by injuries, treatments or training) and correlations to other metrics of clinical impairment known to be prognostic of outcomes may help to improve utility and implementation of force estimation within clinical gait analysis.

\section{APPLICABILITY OF CURRENT MUSCLE FORCE ESTIMATION TECHNIQUES}

\section{TO CLINICAL GAIT ANALYSIS: RECOMMENDATIONS FOR FUTURE}

\section{$\underline{\text { RESEARCH }}$}

The wide range of papers yielded by the search suggests that estimation of muscle force modelling is a practical technique for healthy participants. Processing times [15 minutes for CMC, a few seconds for static optimisation on a "standard" personal computer; 52] are no longer a barrier to clinical application.

There is general agreement in the broad pattern of activity in most muscles (particularly in when in the gait cycle they are on or off) which is also in broad agreement with EMG measurements. (Techniques giving outputs at variance with this broad consensus should, however, now be treated with considerable scepticism.) Differences from this consensus in data from patients might provide information to augment conventional clinical outputs such as joint kinematics and kinetics. Clinical interpretation of these new outputs will have to be based upon an in depth understanding of their biomechanical basis and consequent limitations. This is similar to the manner in which some clinical centres currently incorporate estimates of muscle tendon unit length in their interpretive processes. 
Part of this consideration must be that the underlying assumptions of modelling and simulation are inconsistent with the pathology. Many techniques assume an optimisation of some aspect of neuromuscular function whereas most clinical gait analysis is of people in whom the body's own optimiser, the central nervous system, is deficient in some way. Exploring the applicability of such techniques to musculoskeletal pathologies, in which this is less of an issue, may thus be more appropriate than in neuromuscular pathologies.

Furthermore, musculoskeletal modelling must distinguish between adults, children, and elderly people, as the neuromuscular and skeletal system changes considerable according to age. Further research should systematically review musculoskeletal modelling according to pathology and age.

There is considerable variability between techniques in the patterns of activation and the magnitude of the forces generated and the reasons for this are not clear. According to the results of this review, none of the included techniques can be recommended to be preferential over the others. More research is needed to prove, firstly, the potential advantages of each technique (e.g. activation-contraction cycle in forward dynamic methods) and, secondly, the utility of muscle force estimation in clinical gait analysis (e.g. sensitivity to changes caused by injuries, treatments etc.). For the moment, any clinical implementation should thus focus on that of a single technique in comparison only with normative reference data from that technique.

Force estimations are known to be highly sensitive to model parameters leading to a widely held assumption that person specific models are preferable to generic models. This needs to be balanced by the difficulty in estimating many of the parameters which introduces considerable uncertainty as to how differences from reference data should be interpreted (do differences between patients and reference data occur principally due to differences in individualised model parameters or due to a pathologic change in movement pattern?). 
Some scaling of models is essential, but it might be that limited and systematic scaling leads to data that can be more easily interpreted than fully customised models.

In summary, this review provides some justification for exploratory clinical use of muscle force estimation techniques. It is likely to be more useful in musculoskeletal than neuromuscular conditions and particularly in individuals with fairly normal musculoskeletal anatomy but an abnormal gait pattern.

\section{Conflict of interest statement}

All authors had no financial and personal relationships with other people or organisations that could inappropriately influence (bias) this work.

[1] Herzog W. Muscle. In: Nigg BM, Herzog W, editors. Biomechanics of the Musculoskeletal System. West Sussex, UK: John Wiley \& Sons Ltd.; 1998. p. 148-88.

[2] Yavuz SU, Sendemir-Urkmez A, Turker KS. Effect of gender, age, fatigue and contraction level on electromechanical delay. Clin Neurophysiol. 2010;121:1700-6.

[3] Lin YC, Dorn TW, Schache AG, Pandy MG. Comparison of different methods for estimating muscle forces in human movement. Proceedings of the Institution of Mechanical Engineers Part H - Journal of Engineering in Medicine. 2012;226:103-12.

[4] Arnold AS, Anderson FC, Pandy MG, Delp SL. Muscular contributions to hip and knee extension during the single limb stance phase of normal gait: a framework for investigating the causes of crouch gait. Journal of biomechanics. 2005;38:2181-9.

[5] Anderson FC, Pandy MG. A dynamic optimization solution for vertical jumping in three dimensions. Comput Meth Biomech Biomed Eng. 1999;2:201-31. 
[6] Hatze H. A comprehensive model for human motion sumulation and its application to the take-off phase of the long jump. Journal of biomechanics. 1981;14:135-42.

[7] Erdemir A, McLean S, Herzog W, van den Bogert AJ. Model-based estimation of muscle forces exerted during movements. Clinical Biomechanics. 2007;22:131-54.

[8] Anderson FC, Pandy MG. Static and dynamic optimization solutions for gait are practically equivalent. Journal of biomechanics. 2001;34:153-61.

[9] Pandy MG, Berme N. A numerical method for simulating the dynamics of human walking. Journal of Biomechancis. 1988;21:1043-51.

[10] Delp SL. Surgery Simulation: A Computer Graphics System to Analyse and Design Musculoskeletal Reconstructions of the Lower Limb [Ph.D.]. Stanford, CA: Standford University; 1990.

[11] Higgins J, Green S. Cochrane Handbook for Systematic Reviews of Interventions. 5.1.0 [updated March 2011] ed: The Cochrance Collaboration; 2011.

[12] Winter DA, Patla AE, Frank JS, Walt SE. Biomechanical walking pattern changes in the fit and healthy elderly. Physical Therapy. 1990;70:340-7.

[13] Izquierdo M, Aguado X, Gonzalez RV, López JL, Häkkinen K. Maximal and explosive force production capacity and balance performance in men of different ages. European journal of applied physiology. 1999;79:260-7.

[14] Hughes VA, Frontera WR, Wood M, Evans WJ, Dallal GE, Roubenoff R, et al. Longitudinal Muscle Strength Changes in Older Adults: Influence of Muscle Mass, Physical Activity, and Health. Journal of Gerontology. 2001;56A:B209-B17.

[15] Lee SJ, Hidler J. Biomechanics of overground vs. treadmill walking in healthy individuals. J Appl Physiol. 2008;104:747-55. 
[16] Parvataneni K, Ploeg L, Olney SJ, Brouwer B. Kinematic, kinetic and metabolic parameters of treadmill versus overground walking in healthy older adults. Clinical Biomechanics. 2009;24:95-100.

[17] Liu MQ, Anderson FC, Pandy MG, Delp SL. Muscles that support the body also modulate forward progression during walking. Journal of biomechanics. 2006;39:2623-30. [18] Perry J, Burnfield JM. Gait Analysis: Normal and Pathological Function. 2nd edition: SLACK incorporated; 2010.

[19] Peters A, Galna B, Sangeux M, Morris M, Baker R. Quantification of soft tissue artifact in lower limb human motion analysis: A systematic review. Gait \& posture. 2010;31:1-8. [20] Downs SH, Black N. The feasibility of creating a checklist for the assessment of the methodological quality both of randomised and non-randomised studies of health care interventions. J Epidemiol Community Health. 1998;52:377-284.

[21] Ridgewell E, Dobson F, Bach TM, Baker R. A systematic review to determine best practice reporting guidelines for AFO interventions in studies involving children with cerebral palsy. Prothetics and Orthotics International. 2010;34:129-45.

[22] Fraysse F, Dumas R, Cheze L, Wang X. Comparison of global and joint-to-joint methods for estimating the hip joint load and the muscle forces during walking. Journal of biomechanics. 2009;42:2357-62.

[23] Bazett-Jones DM, Cobb SC, Joshi MN, Cashin SE, Earl JE. Normalizing hip muscle strength: establishing body-size-independent measurements. Arch Phys Med Rehabil. 2011;92:76-82.

[24] Kadaba MP, Ramakrishnen HK, Wootten JG, Gainey J, Gorton G, Cochran GVB. Repeatability of Kinematic, Kinetic, and Electromyographic Data in Normal Adult Gait. Journal Of Orthopaedic Research. 1989;7:849-60. 
[25] Buchanan TS, Lloyd DG, Manal K, Besier TF. Estimation of Muscle Forces and Joint Moments Using a Forward-Inverse Dynamics Model. Medicine \& Science in Sports \& Exercise. 2005;37:1911-6.

[26] De Groote F, Pipeleers G, Jonkers I, Demeulenaere B, Patten C, Swevers J, et al. A physiology based inverse dynamic analysis of human gait: potential and perspectives. Computer Methods in Biomechanics \& Biomedical Engineering. 2009;12:563-74.

[27] Hase K, Yamazaki N. Development of a Three-Dimensional Whole Body Musculoskeletal Model for Various Motion Analysis. Jsme International Journal Series CDynamics Control Robotics Design and Manufacturing. 1997;40:25-32.

[28] Leardini A, O'Connor JJ. A model for lever-arm length calculation of the flexor and extensor muscles at the ankle. Gait \& posture. 2002;15:220.

[29] White SC, Winter DA. Predicting Muscle Forces in Gait from EMG Signals and Musculotendon Kinematics. Journal of Electromyography and Kinesiology. 1993;2:217-31. [30] Bogey RA, Gitter AJ, Barnes LA. Determination of ankle muscle power in normal gait using an EMG-to-force processing approach. Journal of Electromyography and Kinesiology. 2010;20:46-54.

[31] Rodrigo SE, Ambrósio JAC, Tavares da Silva MP, Penisi OH. Analysis of Human Gait Based on Multibody Formulations and Optimization Tools. Mechanics Based Design of Structures and Machines. 2008;36:446-77.

[32] Thompson JA, Chaudhari AM, Schmitt LC, Best TM, Siston RA. Gluteus maximus and soleus compensate for simulated quadriceps atrophy and activation failure during walking. Journal of biomechanics. 2013;46:2165-72.

[33] Valente G, Taddei F, Jonkers I. Influence of weak hip abductor muscles on joint contact forces during normal walking: probabilistic modeling analysis. Journal of biomechanics. 2013;46:2186-93. 
[34] Delp SL, Loan P, Hoy MG, Zajac FE, Topp EL, Rosen JM. An interactive graphicsbased model of the lower extremtiy to study orhtopaedics surgical procedures. IEEE transactions on bio-medical engineering. 1990;37:757-67.

[35] Zajac FE. Muscle and Tendon: Properties, Models, Scaling, and Application to Biomechanics and Motor Control. Critical Reviews in Biotechnology. 1989;17:359-411. [36] Besier T, Fredericson M, Gold C, Beaupre G, Delp SL. Knee muscle forces during walking and running in patellofemoral pain patients and pain-free controls. Journal of biomechanics. 2009;42:898-905.

[37] Ackland DC, Lin YC, Pandy MG. Sensitivity of model predictions of muscle function to changes in moment arms and muscle-tendon properties: a Monte-Carlo analysis. Journal of biomechanics. 2012;45:1463-71.

[38] Lim YP, Lin YC, Pandy MG. Muscle function during gait is invariant to age when walking speed is controlled. Gait \& posture. 2013;38:253-9.

[39] Dempster W. Space Requirements of the Seated Operator. Geometrical, Kinematic, and Mechanical Aspects of the BodyWith Special Reference to the Limbs. Wright-Patterson Air Force Base, OH: Wright Air Development Center, Air Research and Development Command; 1955.

[40] Chandler R, Clauser C, McConville J, Reynolds H, Young J. Investigation of the inertial properties of the human body. Springfield, Virgina1975.

[41] Havanan E. A Mathematical Model of the Human Body. Ohio: Aerospace Medical Division; 1964. p. 64-102.

[42] Zatsiorsky V, Seluyanov V, Chugunova L. Methonds of determining mass-inertia characteristics of human body segments. In: Chernyi G, Regirer S, editors. Contemporary Problems of Biomechanics. Massachusetts: CRC Press; 1990. p. 272-91. 
[43] McConville J, Clauser C, Churchill T, Cuzzi JR, Kaleps I. Anthropometric relationships of bod yand body segment moments of inertia. Wright-Patterson AFB, Ohio: Air Force Aerospace Medical Research Laboratory; 1980.

[44] Inman V. The Pattern of Muscular Activity in the Lower Extremity during Walking. University of California; 1953.

[45] Perry J. Gait nalysis: Normal and Pathological Function. Thorofare, NJ: SLACK Incorporated; 1992.

[46] Hof AL, Elzinga H, Grimmius W, Halbertsma JPK. Speed dependence of averaged EMG profiles in walking. Gait \& posture. 2002;16:78-86.

[47] Heintz S, Gutierrez-Farewik EM. Static optimization of muscle forces during gait in comparison to EMG-to-force processing approach. Gait \& Posture. 2007;26:279-88.

[48] Collins JJ. The redundant nature of locomotor optimization laws. Journal of biomechanics. 1995;28:251-67.

[49] Pinzone O, Schwartz MH, Baker R. Comprehensive non-dimensional normalization of gait data. Gait Posture. 2016;44:68-73.

[50] Hicks JL, Uchida TK, Seth A, Rajagopal A, Delp S. Is my model good enough? Best practices for verification and validation of musculoskeletal models and simulations of human movement. J Biomech Eng. 2014.

[51] Kinney AL, Besier TF, D'Lima DD, Fregly BJ. Update on grand challenge competition to predict in vivo knee loads. Journal of biomechanical engineering. 2013;135:021012. [52] De Groote F, Demeulenaere B, Swevers J, De Schutter J, Jonkers I. A physiology-based inverse dynamic analysis of human gait using sequential convex programming: a comparative study. Comput Methods Biomech Biomed Engin. 2012;15:1093-102. 
[53] Bogey RA, Perry J, Gitter AJ. An EMG-to-Force Processing Approach for Determining Ankle Muscle Forces During Normal Human Gait. IEEE Transactions on Neural Systems and Rehabilitation Engineering. 2005;13:302-10.

[54] Glitsch U, Baumann W. The three-dimensional determination of internal loads in the lower extremity. Journal of biomechanics. 1997;30:1123-31.

[55] Komura T, Nagano A. Evaluation of the influence of muscle deactivation on other muscles and joints during gait motion. Journal of biomechanics. 2004;37:425-36.

[56] Komura T, Nagano A, Leung H, Shinagawa Y. Simulating pathological gait using the enhanced linear inverted pendulum model. IEEE Transactions on Biomedical Engineering. 2005;52:1502-13.

[57] Silva MPT, Ambrosio JAC. Solution of redundant muscle forces, in human locomotion with multibody dynamics and optimization tools. Mechanics Based Design of Structures and Machines. 2003;31:381-411. 
Table 1. Relevant studies included into the systematic review process; $\mathrm{SO}=$ static optimisation, $\mathrm{FD}=$ forward dynamics, $\mathrm{ED}=\mathrm{EMG}$-driven, $\mathrm{ID}=$ inverse dynamics, $\mathrm{CMC}=$ computed muscle control, $\mathrm{a}=$ activation, $\mathrm{e}=$ excitations, $\mathrm{f}=$ force, $\mathrm{s}=$ stress, $\mathrm{BC}=$ Bertec Corporation, $\mathrm{MAC}=$ Motion Analysis Corporation, MLS=Motion Lab System, $\mathrm{S}=$ stance, $\mathrm{GC}=$ gait cycle.

\begin{tabular}{|c|c|c|c|c|}
\hline Authors [ID] & $\begin{array}{l}\text { Mathematical } \\
\text { model (model } \\
\text { (cost function)) }\end{array}$ & $\begin{array}{c}\text { Measurement } \\
\text { equipment (motion system } \\
(H z), \text { force plates }(H z), E M G \\
(H z)) \\
\end{array}$ & $\begin{array}{l}\text { Gait }(\mathrm{m} / \mathrm{s} \\
\text { stance or } \\
\quad G C)\end{array}$ & $\begin{array}{c}\text { Participants } \\
(\text { number, } B W \\
(\mathrm{kg}), B H(m), \text { age })\end{array}$ \\
\hline Ackland et al. [37] & $\mathrm{SO}\left(\sum \mathrm{a}^{2}\right)$ & Vicon (120), --, // & free, $S$ & $1,70,1.78,26$ \\
\hline $\begin{array}{r}\text { Anderson \& Pandy } \\
{[8]}\end{array}$ & $\begin{array}{l}\mathrm{SO}\left(\sum \mathrm{a}^{2}\right) \\
\mathrm{FD}(\text { metab. energy })\end{array}$ &,,$---- / /$ & $1.12, \mathrm{GC}$ & $1,70.1,1.77,26$ \\
\hline Besier et al. [36] & $\mathrm{ED}\left(\sum\right.$ moments $)$ & $\begin{array}{l}\text { MAC (60), BC (2400), MLS } \\
(2400)\end{array}$ & $1.49, \mathrm{~S}$ & $16,74.2,1.79,28$ \\
\hline Bogey et al. [53] & $\mathrm{ED}\left(\sum\right.$ moments $)$ & $\begin{array}{l}\text { Vicon (100), Kistler (600), } \\
\text { needle }\end{array}$ & 1.37, GC & $10,76,1.78,27$ \\
\hline Bogey et al. [30] & ED ( $($ power $)$ & $\begin{array}{l}\text { MAC (100), AMTI (600), } \\
\text { needle (2500) }\end{array}$ & free, $\mathrm{GC}$ & $16,73.9,--, 27$ \\
\hline Buchanan et at. [25] & $\mathrm{ED}\left(\sum\right.$ moments $)$ & $\begin{array}{l}\text { Qualysis (--), AMTI (--), } \\
\text { Noraxon (1000) }\end{array}$ & free, $S$ & $1,--,--,--$ \\
\hline Collins et al. [48] & $\mathrm{SO}\left(\sum \mathrm{f}\right)$ & Vicon (50), Kistler (50), // &,$-- \mathrm{GC}$ & $1,69.8,1.76,26$ \\
\hline DeGroote et al. [26] & $\begin{array}{l}\mathrm{SO}\left(\sum \mathrm{a}^{2}\right) \\
\text { physical ID }\left(\sum \mathrm{a}^{2}\right)\end{array}$ & Qualysis (--), AMTI (--),// &,-- GC & $1,--,--,--$ \\
\hline Fraysse et at. [22] & $\mathrm{SO}\left(\sum \mathrm{s}^{2}\right)$ & MAC (100), AMTI, (100) // & free, $\mathrm{GC}$ & $1,64,1.74,23$ \\
\hline $\begin{array}{r}\text { Glitsch \& Baumann } \\
\text { [54] }\end{array}$ & $\mathrm{SO}\left(\sum \mathrm{s}^{2}\right)$ & $\begin{array}{l}\text { Selspot II (500), Kistler } \\
(500), / /\end{array}$ & $1.5, \mathrm{~S}$ & $1,85,1.78,31$ \\
\hline $\begin{array}{r}\text { Hase \& Yamazaki } \\
\text { [27] }\end{array}$ & FD (metab. energy) & $\begin{array}{l}\text { Hamasatsu Photonics PSD } \\
\text { system C3570 (--), Kyowa } \\
\text { Dengyo EP-386 (--), // }\end{array}$ & $1.39, \mathrm{GC}$ & $1,--,--,--$ \\
\hline $\begin{array}{r}\text { Heintz \& Gutierrez- } \\
\text { Farewik [47] }\end{array}$ & $\begin{array}{l}\mathrm{SO}\left(\sum \mathrm{s}^{2}\right) \\
\mathrm{ED}\left(\sum \mathrm{moments}\right)\end{array}$ & $\begin{array}{l}\text { Vicon (50), Kistler (--), } \\
\text { MLS (1000) }\end{array}$ & free, GC & $1,59.3,1.64,34$ \\
\hline $\begin{array}{r}\text { Komura \& Nagano } \\
\text { [55] }\end{array}$ & $\mathrm{SO}\left(\sum \mathrm{s}\right)$ & Vicon $(--),--, / /$ &,$-- \mathrm{S}$ & $1,51,1.64,50$ \\
\hline Komura et al. [56] & $\mathrm{SO}\left(\sum \mathrm{s}^{2}\right)$ &,,$---- / /$ & $1.0, \mathrm{GC}$ & $1,51,1.64,50$ \\
\hline $\begin{array}{r}\text { Leardini \& O'Connor } \\
\text { [28] }\end{array}$ & SO (--) & $\begin{array}{l}\text { ElitePlus BTS (--), Kistler (- } \\
-), / /\end{array}$ &,$-- S$ & $1,59,1.74,--$ \\
\hline Lim et al. [38] & $\mathrm{SO}\left(\sum \mathrm{a}^{2}\right)$ & Vicon (120), AMTI (1080) & $1.4, \mathrm{~S}$ & $10,65.9,1.70,25$ \\
\hline Lin et al. [3] & $\begin{array}{l}\operatorname{SO}\left(\sum \mathrm{a}^{2}\right) \\
\operatorname{FD}\left(\sum \mathrm{a}^{2}+\sum \text { errors }\right) \\
\operatorname{CMC}\left(\sum \mathrm{a}^{2}\right)\end{array}$ & Vicon (250), Kistler (--), // & $1.61, \mathrm{GC}$ & $1,64,1.77,25$ \\
\hline Rodrigo et al. [31] & SO (metab. energy) &,,$---- / /$ & $\begin{array}{l}111 \mathrm{st} / \mathrm{min}, \\
\mathrm{GC}\end{array}$ & $1,70,1.70,25$ \\
\hline $\begin{array}{r}\text { Silvia \& Ambrosio } \\
\text { [57] }\end{array}$ & $\mathrm{SO}\left(\sum \mathrm{s}\right)$ &,,---- /I & free, $\mathrm{GC}$ & $1,70,1.70,25$ \\
\hline Thompson et al. [32] & $\mathrm{CMC}\left(\sum \mathrm{e}^{2}\right)$ & VICON (150), Bertec (600) & $1.32, \mathrm{GC}$ & $7,72.8,1.74,22$ \\
\hline Valente et al. [33] & CMC (--) & VICON (100), AMTI (1000) & free, GC & $5,82,1.82,26$ \\
\hline White \& Winter [29] & $\mathrm{ED}$ ( $\sum$ moments $)$ & $\begin{array}{l}\text { Bolex }(50+200) \text {, AMTI } \\
(1000),--(500)\end{array}$ &,$-- S$ & $1,--,--, 28$ \\
\hline
\end{tabular}


Table 2. Individual (for each study) and average (across all studies of the same modelling approach) quality scores in percentage [\%].

\begin{tabular}{|c|c|c|c|c|c|c|c|c|c|c|c|c|c|}
\hline Authors [ID] & Aim & $\begin{array}{c}\text { Partici- } \\
\text { pants }\end{array}$ & Task & $\begin{array}{c}\text { Equip- } \\
\text { ment }\end{array}$ & $\begin{array}{c}\text { Proces- } \\
\text { sing }\end{array}$ & $\begin{array}{c}\text { Kinema- } \\
\text { tic } \\
\end{array}$ & Kinetic & Output & $\begin{array}{c}\text { Valida- } \\
\text { tion }\end{array}$ & $\begin{array}{l}\text { Muscle } \\
\text { Models }\end{array}$ & $E M G$ & $\begin{array}{l}\text { Discus- } \\
\text { sion }\end{array}$ & $\begin{array}{c}\text { Overall } \\
\text { score }\end{array}$ \\
\hline Ackland et al. [37] & 100 & 80 & 25 & 67 & 100 & 43 & 67 & 100 & 0 & 100 & --- & 50 & 67 \\
\hline Anderson \& Pandy [8] & 100 & 33 & 0 & 0 & 0 & 43 & 67 & 50 & 100 & 100 & 0 & 100 & 49 \\
\hline Besier et al. [36] & 100 & 80 & 75 & 67 & 100 & 100 & 60 & 100 & 0 & 100 & 94 & 100 & 81 \\
\hline Bogey et al. [53] & 100 & 60 & 100 & 83 & 100 & 29 & 67 & 50 & 0 & 100 & 63 & 100 & 71 \\
\hline Bogey et al. [30] & 100 & 60 & 100 & 92 & 75 & 29 & 67 & 50 & 50 & 100 & 63 & 50 & 70 \\
\hline Buchanan et at. [25] & 100 & 17 & 25 & 50 & 0 & 14 & 33 & 50 & 100 & 100 & 100 & 100 & 57 \\
\hline Collins et al. [48] & 100 & 33 & 25 & 50 & 0 & 57 & 67 & 50 & 100 & 80 & 25 & 50 & 53 \\
\hline DeGroote et al. [26] & 100 & 17 & 0 & 33 & 0 & 75 & 100 & 50 & 100 & 100 & 25 & 100 & 58 \\
\hline Fraysse et at. [22] & 100 & 80 & 25 & 67 & 50 & 71 & 67 & 75 & 0 & 100 & --- & 100 & 67 \\
\hline Glitsch \& Baumann [54] & 100 & 83 & 100 & 92 & 0 & 29 & 100 & 50 & 100 & 80 & 75 & 100 & 76 \\
\hline Hase \& Yamazaki [27] & 100 & 17 & 0 & 33 & 0 & 43 & 33 & 50 & 50 & 100 & --- & 100 & 48 \\
\hline Heintz \& Gutierrez-Farewik [47] & 100 & 100 & 33 & 50 & 0 & 86 & 67 & 50 & 100 & 100 & 88 & 100 & 73 \\
\hline Komura \& Nagano [55] & 100 & 100 & 0 & 17 & 0 & 43 & 67 & 50 & 50 & 100 & --- & 100 & 57 \\
\hline Komura et al. [56] & 100 & 100 & 0 & 17 & 0 & 80 & 67 & 50 & 50 & 100 & --- & 0 & 51 \\
\hline Leardini \& O'Connor [28] & 100 & 67 & 0 & 17 & 0 & 86 & 0 & 50 & 0 & 100 & 63 & 100 & 49 \\
\hline Lim et al. [38] & 100 & 90 & 67 & 83 & 50 & 75 & 33 & 100 & 100 & 100 & 50 & 100 & 77 \\
\hline Lin et al. [3] & 100 & 100 & 67 & 50 & 100 & 57 & 60 & 50 & 100 & 100 & 25 & 100 & 76 \\
\hline Rodrigo et al. [31] & 100 & 100 & 67 & 50 & 100 & 100 & 100 & 50 & 0 & 100 & --- & 100 & 72 \\
\hline Silvia \& Ambrosio [57] & 100 & 83 & 33 & 50 & 0 & 71 & 100 & 50 & 0 & 100 & --- & 100 & 62 \\
\hline Thompson et al. [32] & 100 & 100 & 75 & 100 & 0 & 75 & --- & 100 & 100 & 80 & 100 & 100 & 81 \\
\hline Valente et al. [33] & 100 & 100 & 25 & 67 & 25 & 63 & --- & 100 & 100 & 60 & 75 & 100 & 71 \\
\hline White \& Winter [29] & 100 & 33 & 25 & 92 & 50 & 43 & 67 & 50 & 100 & 100 & 50 & 100 & 68 \\
\hline Static Optimisation & 100 & 76 & 32 & 46 & 29 & 65 & 69 & 59 & 57 & 97 & 44 & 86 & $63 \pm 11$ \\
\hline Forward Dynamics & 100 & 50 & 22 & 28 & 33 & 48 & 53 & 50 & 83 & 100 & 13 & 100 & $58 \pm 16$ \\
\hline EMG-driven & 100 & 58 & 60 & 72 & 54 & 50 & 60 & 58 & 58 & 100 & 76 & 92 & $70 \pm 8$ \\
\hline Enhanced SO & 100 & 95 & 48 & 71 & 44 & 67 & 64 & 81 & 75 & 85 & 67 & 100 & $74 \pm 6$ \\
\hline Average & 100 & 70 & 39 & 56 & 34 & 60 & 64 & 63 & 59 & 95 & 60 & 89 & $65 \pm 11$ \\
\hline
\end{tabular}


Table 3. Average and standard deviation of peak muscle force with coefficient of variation (expressed as a \%) and average timing of the peak within the gait cycle for stance and swing; $\mathrm{GC}=$ gait cycle, $\mathrm{SD}=$ standard deviation, $\mathrm{FD}=$ forward dynamics, $\mathrm{SO}=$ static optimisation, $\mathrm{CV}=$ coefficient of variation, EMG-dr.= EMG-driven

\begin{tabular}{|c|c|c|c|c|c|c|c|c|c|}
\hline \multirow[b]{2}{*}{ Muscle } & \multirow[b]{2}{*}{ Modelling approach } & \multicolumn{4}{|c|}{ Maximum } & \multicolumn{4}{|c|}{ Maximum } \\
\hline & & Average & SD & $\begin{array}{l}\text { CV } \\
(\%)\end{array}$ & $\begin{array}{l}\text { GC } \\
(\%)\end{array}$ & Average & SD & $\begin{array}{l}\text { CV } \\
(\%)\end{array}$ & $\begin{array}{l}\text { GC } \\
(\%)\end{array}$ \\
\hline \multirow{4}{*}{$\begin{array}{l}\text { Tibialis } \\
\text { anterior }\end{array}$} & SO (7) & 5.6 & 4.3 & 78 & 6 & 2.6 & 1.7 & 63 & 96 \\
\hline & Enhanced-SO (1) & 4.4 & -- & -- & 0 & 4.5 & -- & -- & 65 \\
\hline & FD (1) & -- & -- & -- & -- & 1.1 & -- & -- & 65 \\
\hline & EMG-dr. (3) & 3.0 & 2.8 & 94 & 4 & 2.8 & 1.0 & 35 & 78 \\
\hline \multirow{4}{*}{$\begin{array}{l}\text { Gastroc- } \\
\text { nemius }\end{array}$} & SO (11) & 14.8 & 7.1 & 48 & 44 & & & & \\
\hline & Enhanced-SO (2) & 14.9 & 3.0 & 20 & 45 & & & & \\
\hline & $\mathrm{FD}(2)$ & 12.4 & 0.9 & 8 & 45 & & & & \\
\hline & EMG-dr. (4) & 13.5 & 2.9 & 21 & 46 & & & & \\
\hline \multirow[t]{4}{*}{ Soleus } & SO (12) & 20.0 & 9.7 & 48 & 47 & & & & \\
\hline & Enhanced-SO (4) & 18.5 & 4.0 & 22 & 52 & & & & \\
\hline & FD (2) & 23.1 & 1.6 & 7 & 48 & & & & \\
\hline & EMG-dr. (5) & 16.5 & 7.8 & 47 & 46 & & & & \\
\hline \multirow{4}{*}{$\begin{array}{l}\text { Rectus } \\
\text { femoris }\end{array}$} & SO (9) & 2.7 & 1.6 & 58 & 21 & 4.2 & 2.4 & 57 & 54 \\
\hline & Enhanced-SO (3) & 2.6 & 2.0 & 76 & 5 & 4.4 & 1.7 & 38 & 52 \\
\hline & FD (3) & 2.3 & 2.8 & 120 & 10 & 5.9 & 7.8 & 133 & 51 \\
\hline & EMG-dr. (2) & 1.7 & 1.4 & 80 & 12 & 1.9 & -- & -- & 63 \\
\hline \multirow[t]{4}{*}{ Vastii } & SO (9) & 13.3 & 9.3 & 70 & 12 & 3.1 & 2.3 & 75 & 62 \\
\hline & Enhanced-SO (4) & 8.7 & 2.7 & 30 & 13 & 3.4 & 1.2 & 37 & 69 \\
\hline & $\mathrm{FD}(2)$ & 14.1 & 3.4 & 24 & 12 & 5.2 & 0.8 & 16 & 62 \\
\hline & EMG-dr. (2) & 10.3 & 7.0 & 68 & 13 & -- & -- & -- & -- \\
\hline \multirow[t]{4}{*}{ Hamstrings } & SO (9) & 7.2 & 5.3 & 73 & 2 & 4.6 & 1.7 & 37 & 94 \\
\hline & Enhanced-SO (1) & 6.0 & -- & -- & 3 & 2.8 & -- & -- & 96 \\
\hline & $\mathrm{FD}(2)$ & 4.0 & 2.4 & 61 & 2 & 3.5 & 0.7 & 21 & 94 \\
\hline & EMG-dr. (2) & 4.1 & 2.6 & 63 & 2 & -- & -- & -- & -- \\
\hline \multirow[t]{3}{*}{ Iliopsoas } & SO (5) & 7.2 & 7.9 & 111 & 47 & & & & \\
\hline & Enhanced-SO (2) & 24.8 & 3.9 & 16 & 49 & & & & \\
\hline & FD (3) & 9.0 & 12.6 & 140 & 50 & & & & \\
\hline \multirow[t]{3}{*}{ Gluteus max } & SO (6) & 4.6 & 3.5 & 78 & 13 & & & & \\
\hline & Enhanced-SO (3) & 3.6 & 1.3 & 37 & 12 & & & & \\
\hline & FD (4) & 4.8 & 4.9 & 102 & 12 & & & & \\
\hline \multirow[t]{3}{*}{ Gluteus med } & SO (7) & 14.7 & 22.2 & 151 & 8 & & & & \\
\hline & Enhanced-SO (2) & 19.1 & 21.3 & 112 & 15 & & & & \\
\hline & FD (2) & 27.9 & 26.4 & 94 & 12 & & & & \\
\hline
\end{tabular}




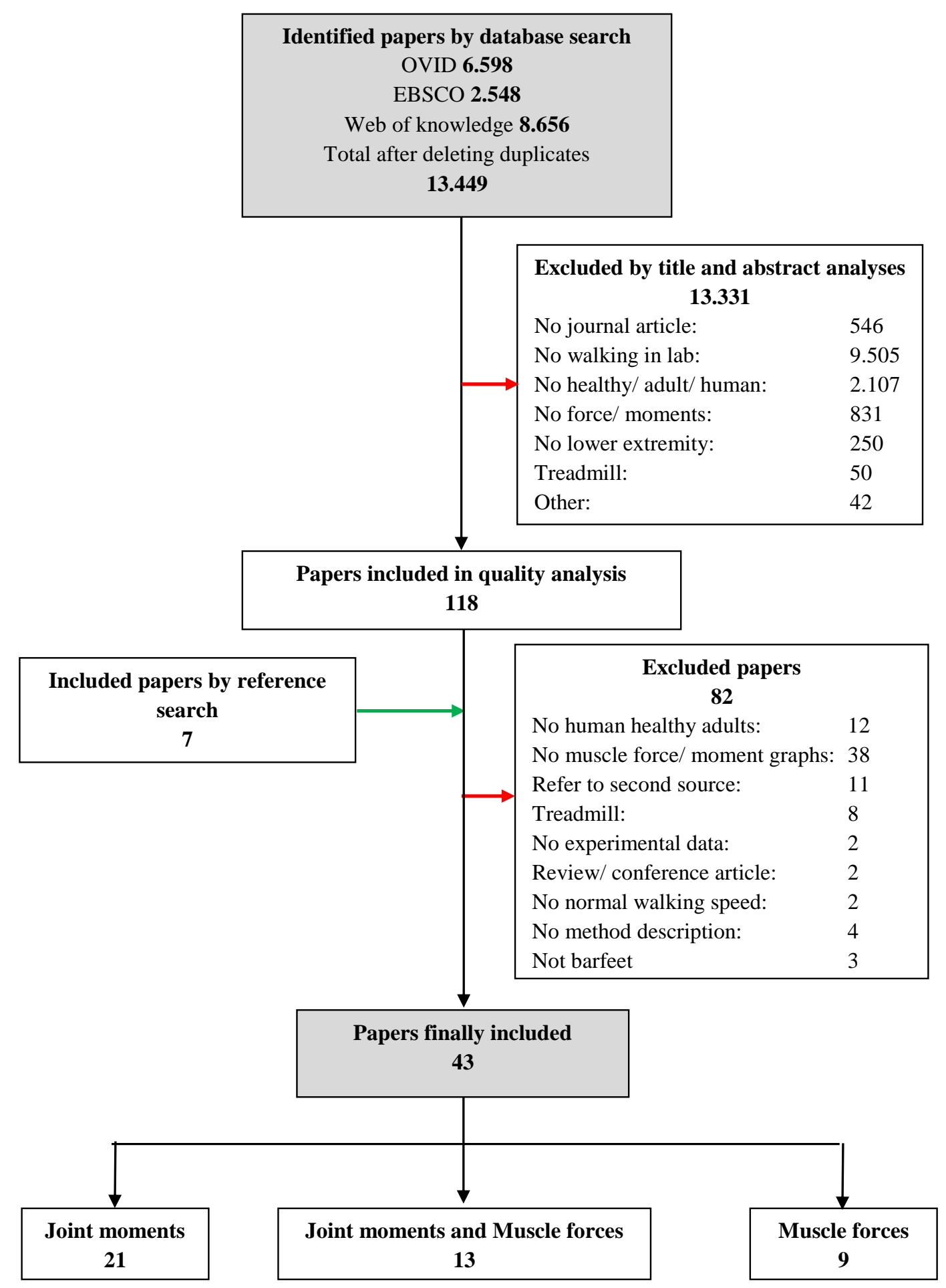

Figure 1. Flow chart describing the process of the systematic review. Red arrows indicate exclusion, green arrows inclusion of papers. "No walking in lab" indicates other movements than walking or other investigation environments other than a movement laboratory. 



Figure 2. Mean and one and two standard deviation of extracted muscle forces of the lower limb during healthy walking divided in static optimisation, EMG-driven models and forward dynamics. Curves of CMC of Lin et al. (black) and physiological inverse dynamics of 
DeGroote et al. (grey) are in the column "other". Forces are shown in Newton/body mass, numbers of included studies are shown in brackets. 